\title{
Competencia argumentativa mediante la investigación como estrategia pedagógica en educación básica ${ }^{1}$
}

\section{Argumentative competence through research as a pedagogical strategy in basic education}

DOI: http://dx.doi.org/10.17981/cultedusoc.9.1.2018.12

Fecha de recepción: 28/04/2018. Fecha de aceptación: 26/07/2018

Elida Esther Flórez-Jassan ${ }^{2}$

Alfredo Said Quinto-Pérez; Alma Bruna Cortes-Peralta; Andrés Augusto Sánchez-Cervantes; Arelis Asceneth Pérez-Charris; Claribeth Contreras-Quiroz; Daniel Mendoza-Fadul;

Doralis Ibeth Jiménez-Herrera; Ernesto Rafael Miranda-Ropain;

Fabián David Caballero-Chacón; Maritza Del Carmen Diazgranados-Corrales; Nelly Sofía Amaris-Villalba; Olga Luz Henríquez-Henríquez ${ }^{3}$

Para citar este artículo

Flórez-Jassan, E., Quinto-Pérez, A., Cortes-Peralta, A., Sánchez-Cervantes, A., Pérez-Charris, A., Contreras-Quiroz, C., Mendoza-Fadul, D., Jiménez-Herrera, D., Miranda-Ropain, E., Caballero-Chacón, F., Diazgranados-Corrales, M., Amaris-Villalba, N. y Henríquez-Henríquez, O. (2018). Competencia argumentativa mediante la investigación como estrategia pedagógica en educación básica. Cultura. Educación y Sociedad 9(1), 160-170. DOI: http://dx.doi.org/10.17981/cultedusoc.9.1.2018.12

\section{Resumen}

Las competencias son capacidades, habilidades y conocimientos que posee una persona para responder a una situación sin limitarse a ella, estas se miden de acuerdo al desempeño con el que se realice, donde las estrategias pedagógicas cumplen un papel determinante en la motivación del estudiante. El estudio busco fortalecer las competencias cognitivas mediante la investigación como estrategia pedagógica en educación básica. Está guiado bajo los lineamientos metodológicos de la IEP con un diseño basado en las trayectorias de indagación, se seleccionó una muestra de cuarenta (40) estudiantes del grado octavo (8) de bachillerato, de la Institución Educativa Departamental Fundación, ubicada en el departamento del Magdalena. Para la recolección de información se utilizó un cuestionario estructurado, mediante una encuesta para valorar las estrategias implementadas apoyadas en la IEP. Los resultados arrojaron diversas estrategias pedagógicas para el desarrollo de las competencias argumentativas mediante la investigación como estrategia pedagógica en la educación básica.

Palabras clave: competencia argumentativa, investigación como estrategia pedagógica, educación básica.

\begin{abstract}
Competencies are abilities, skills and knowledge that a person has to respond to a situation without limiting itself to it, these are measured according to the performance with which it is carried out, where pedagogical strategies play a decisive role in the motivation of the student. The study sought to strengthen cognitive skills through research as a pedagogical strategy in basic education. It is guided under the methodological guidelines of the IEP with a design based on the trajectories of inquiry, a sample of forty (40) eighth grade (8) baccalaureate students was selected from the Departmental Educational Institution Foundation, located in the department of Magdalena For the collection of information, a structured questionnaire was used, through a survey to assess the implemented strategies supported by the IEP. The results showed various pedagogical strategies for the development of argumentative competences through research as a pedagogical strategy in basic education.
\end{abstract}

Keywords: argumentative competence, research as a pedagogical strategy, basic education.

\footnotetext{
${ }^{1}$ Este artículo ha sido derivado del Programa de Fortalecimiento de la Cultura Ciudadana y Democrática CT+I a través de la IEP apoyada en TIC en el Departamento de Magdalena: CICLON

${ }^{2}$ Docente de la Institución Educativa Departamental Colombia y líder del grupo de investigación Estrategas de Colombia. Correo de correspondencia: eflorez2268@hotmail.com

${ }^{3}$ Docentes de la IED Colombia, Sede principal y miembros del grupo de investigación Estrategas de Colombia.
}

The author; licensee Universidad de la Costa - CUC. 


\section{Introducción}

Las instituciones educativas tienen la finalidad de fomentar el aprendizaje de los estudiantes proporcionando una formación integral que abarque competencias necesarias para un óptimo desarrollo social, permitiendo el ejercicio de valores y deberes sustentados en la práctica de una ciudadanía democrática. Sin embargo, el estudiante debe tener un rol activo en la construcción de su conocimiento, desarrollando habilidades prácticas, motivación, valores éticos, actitudes, emociones y otros componentes sociales que se movilizan para lograr un aprendizaje significativo, las habilidades anteriormente mencionadas permiten el desarrollo de competencias cognitivas y argumentativas, que favorecen el pensamiento crítico, reflexivo y abstracto, fortaleciendo así habilidades sociales.

En ese sentido, el rol del docente se transforma, integrando practicas constructivistas, donde realiza un acompañamiento al estudiante, promoviendo el desarrollo de competencias y habilidades para la interiorización y elaboración de su propio conocimiento a través de la formación y el apoyo que brinde el docente, generando practicas pedagógicas que dinamicen el proceso enseñanza aprendizaje.

No obstante, en Colombia aprender a gestionar de forma autónoma este tipo de tareas conlleva una gran complejidad, donde la falta de sincronía y presencia física reduce y dificulta las posibilidades de ponerlas en práctica. A fin de ofrecer algunas alternativas de formación en este sentido, por tal motivo se debe fortalecer las competencias cognitivas mediante la investigación como estrategia pedagógica (IEP) en educación básica.
Argumentar es una actividad cotidiana y necesaria en la vida de todo profesional, sea este abogado, médico, ingeniero, administrador, vendedor, banquero e incluso para un individuo cualquiera. Todo aquel que crea que debe defender con éxito sus ideas o refutar las de otro oponente necesita desarrollar la destreza argumentativa. Por tanto, la formación universitaria exige el desarrollo y el refinamiento de tal competencia.

En este sentido, se realizó un estudio en la Institución Educativa Departamental Colombia, ubicada en el Municipio de Fundación - Magdalena, la cual vela por brindar una educación de calidad a sus estudiantes, por lo cual evalúa en cada periodo académico cuatro (4) aspectos básicos; cognitivo, procedimental, actitudinal y prueba por competencias. Observando que el aspecto cognitivo presenta resultados inferiores, por tal razón surge la necesidad de realizar un análisis para conocer las posibles causas y de esta manera crear estrategias para mejorar dicho aspecto.

A partir de un diagnóstico realizado por el cuerpo docente de la institución, como antecedente a esta investigación, se logró identificar que las dificultades que prevalecen en el área cognitiva son; argumentación, capacidad discursiva, habilidades lectoras, análisis y razonamiento. Siendo el grado octavo el que obtuvo los puntajes más bajos, mostrando dificultades para expresar sus ideas y sus argumentos carecían de coherencia y cohesión. El estudio apunto al fortalecimiento de la competencia argumentativa, puesto que esta presento falencias dentro de las competencias cognitivas. Identificando dificultades para explicar un texto y sustentar actividades que impliquen un nivel básico de razonamiento, por lo anterior el propósito de la investigación fue 
fortalecer la competencia argumentativa mediante la IEP en educación básica.

Los maestros al identificar dichas falencias se agruparon para buscar soluciones desde la academia, mediante estrategias apoyadas en la IEP encaminadas a fortalecer las competencias argumentativas, no obstante, es importante resaltar que la competencia argumentativa, está directamente relacionada con la comprensión lectora, puesto que esta última permite que el estudiante entienda la idea principal del texto y la argumentación consiste en presentar una postura crítica frente al tema.

La propuesta de integrar la IEP al aula favorece el fortalecimiento de competencias argumentativas, ya que a través de la investigación se fortalecen procesos de pensamiento y razonamiento, permitiendo que los estudiantes organicen, expongan y propongan ideas, contribuyendo a la divulgación del conocimiento, la participación, innovación y competencias comunicativas, (Jimenez, 2010).

\section{Competencia argumentativa mediante la IEP}

Las competencias son; capacidades, conocimientos y estrategias que poseen las personas para responder a una situación, estas se miden de acuerdo al nivel de desempeño con el que se realice. Rojas, (2014) las define como herramientas para resolver una tarea en situaciones determinadas a partir del uso de recursos como; saber hacer en contexto, saber hacer, ser y saber-saber. En ese sentido, están ligadas a la actitud y al carácter personal.

En el contexto escolar la idea de crear competencias se asocia con la capacidad para identificar y encontrar los conocimientos pertinentes, para en- frentar una situación. En ese sentido, el desarrollo de competencias en el ámbito escolar, se encuentra relacionado con la posibilidad de potencializar en los estudiantes habilidades como; escritura, lectura y compresión, que favorezcan el desempeño del estudiante en las diferentes áreas del conocimiento, (Alarcón, Cortes, \& Rodríguez, 2016).

Por su parte, las competencias cognitivas son procesos mentales que se relacionan con la capacidad del ser humano para pensar y tomar decisiones; estas hacen parte del pensamiento como proceso superior, permitiendo recibir el conocimiento, almacenarlo, recuperarlo y utilizarlo posteriormente cuando sea necesario. Las habilidades cognitivas son indispensables para que se dé el aprendizaje, por lo cual estas se encuentran inmersas en los planes de estudio de las instituciones de educación básica y en las de educación superior, (Ramos, Herrera, \& Ramírez, 2010).

Para desarrollar una habilidad cognitiva es necesario que se den tres momentos: primero; el desconocimiento de la habilidad, segundo: el proceso de adquisición, desarrollo y adopción de la misma y tercero; se realiza de forma automática luego de estar interiorizada. Las competencias cognitivas se dividen en tres tipos: la competencia interpretativa, la argumentativa y la propositiva, Ramos, Herrera, \& Ramírez, 2010).

En la presente investigación se desarrollarán las competencias argumentativas puesto que los estudiantes presentan dificultades en ellas, para definirlas es necesario expresar que hacen parte de las habilidades cognitivas superiores que promueven un pensamiento crítico incluyendo la habilidad del razonamien- 
to como uno de sus procesos, esta permite al sujeto responder al "como", mientras al argumentar se explica el "por qué" de las cosas, justificando las ideas, mediante la exposición de razones. Posteriormente se establecen criterios y se interactúa con el saber adquirido, (Guzmán, Flores, \& Tirado, 2013).

Así mismo, las competencias argumentativas brindan la capacidad de organización de elementos: como; solución de contradicciones, evaluación de información y reconsideración de afirmaciones, estos elementos mediante el desarrollo de habilidades cognitivas permiten priorizar la interacción social, además, estas son necesarias para negociar, enfrentarse a una discusión o debates de ideas y posturas. Es decir, que la argumentación es un proceso que implica un nivel superior de razonamiento permitiendo realizar análisis y adoptar posturas críticas frente a un tema en particular, (Guzmán, Flores, \& Tirado, 2013).

Argumentar es un proceso mediante el cual las personas sustentan su postura frente a un tema específico, donde no se trata de encontrar la verdad, sino observar de forma crítica analizando la información, para luego exponer si se está de acuerdo o no justificando el porqué de dicha postura. Para lograr este proceso de argumentación es importante realizar previamente un proceso analítico y sintáctico, además, en desarrollar esta competencia debe haber una relación entre lenguaje y pensamiento, ya que de estos elementos depende la capacidad de expresión de manera clara y lógica sobre los conocimientos que se tengan acerca de un tema específico, (Calderón \& Sarmiento, 2014).
Existen diferentes tipos de argumentación, que permiten desarrollar el control de la competencia; argumentación causal, esta pretende razonar sobre alguna situación determinada, esta sería producto de un factor determinante, es decir, estos hechos explican el origen de alguna nueva situación. Otro tipo es el de argumentos basados en principios; este implica el contexto social rescatando principios que brindan la posibilidad de apoyarse en ellos para dar una explicación argumentativa, (Calderón \& Sarmiento, 2014).

Por otro lado, el contraste de ideas es un tipo de competencia argumentativa que compara dos o más ideas contrastando las opiniones que surgen desde las experiencias o hipótesis de una situación. Mientras que los argumentos empíricos son aquellos que sustentan una idea mediante la ejemplificación de una hipótesis empleando casos específicos y reales basados en experiencias. Finalmente, los argumentos de autoridad son otro tipo de competencia argumentativa, que son emitidos por personas o entidades reconocidas con cierto prestigio entregando información real y comprobable, (Calderón \& Sarmiento, 2014).

Por otro lado, el entorno educativo, es un sistema organizado que permite un nivel de interacción entre estudiantes, docentes y cuerpo administrativo, sin embargo, desde los cursos iniciales de básica primaria, se puede observar un nivel un nivel de comunicación primitivo como señas, gestos y grafos, que posibilita reconocer al otro a través de la escucha atenta junto a la percepción visual, encontrando la información necesaria para motivarlo a aprender el sis- 
tema estructurado de la lengua escrita y hablada, en ese sentido es necesario estimular el aprendizaje de la lectura y escritura en los primeros años, puesto que la curiosidad por desarrollarlos favorece el proceso de enseñanza, para esto utilizar la mediación de las tecnologías de la información puede ser una estrategia didáctica que permita la adquisición de habilidades en lectura y escritura. (Franco, 2010; Marín, Niebles, Sarmiento, \& Valvuena, 2017).

Actualmente existen diversas propuestas basadas en investigación que tienen como objetivo materializar las acciones empleadas en las instituciones educativas, donde a partir de su implementación, surgen propuestas metodológicas para el desarrollo de las clases. En el caso de las pedagogías fundadas en investigación, estas asumen caminos variados en la coherencia de los paradigmas y corrientes puesto que se desarrollan en diferentes contextos; dentro de las pedagogías basadas en investigación, se encuentra la enseñanza por descubrimiento y el programa ondas con su propuesta; investigación como estrategia pedagógica (IEP) esta última retoma para su desarrollo las perspectivas de la pedagogía critico-liberadora y del enfoque socio-cultural, (Mejía \& Manjarrés, 2010).

La propuesta metodológica de la IEP fue desarrollada en Inglaterra y EEUU en los años ochenta por su precursor Jerome Bruner luego esta se extendió a España donde fue adoptada por el profesorado de la Universidad de Valencia para su aplicación, su punto de partida es buscar que los estudiantes desarrollen la capacidad de reconocer un problema como primera tarea. Donde a partir de este, se defina la pregunta problematizadora, que permitirá diseñar y aplicar estrategias que respondan a la situación planteada, logrando así que ellos mediante la reflexión de la práctica investigativa logren construir su propio conocimiento, descubrir la estructura de la realidad, así como teorías, procesos, modelos y productos de la ciencia. Teniendo en cuenta lo anterior podemos inferir que hablamos sobre un proceso de tipo inductivo que parte de lo particular a lo general mediante una guía, (Mejía \& Manjarrés, 2010).

En la implementación de la IEP los contenidos educativos se convierten en problemáticas de posible abordaje y el currículo pasa a estructurarse a partir del surgimiento de una serie de preguntas educativas expuestas por los estudiantes, el avance y desarrollo de los mismos se manifiesta a través del lenguaje, razonamiento y conocimiento que ellos demuestren; ese descubrimiento generalmente no se hace de manera autónoma, es guiado por el docente que se encarga de planificar las estrategias, los ejercicios y los procedimientos para el fin búsqueda, (Mejía \& Manjarrés, 2010).

$\mathrm{El}$ aprendizaje mediante la investigación como estrategia pedagógica IEP está fundamentado a partir de 8 principiaos básicos: Capacidad para resolver problemas como meta principal de la educación; Entrenamiento en nuevas fuentes de conocimiento denominado heurística es más importante que la entrega de conocimientos sin significado a los estudiantes; Cuando el aprendizaje se hace por descubrimiento el estudiante le da más valor, considerándose el mejor camino para enseñar los contenidos curriculares; El aprendizaje 
por descubrimiento logra facilitar una mejor organización de la información aprendida y la guarda de manera eficaz; El dialogo de saberes para promover la trasferencia de conocimiento; El aprendizaje por descubrimiento que promueve la confianza y el desarrollo de pensamiento abstracto; El descubrimiento permite la asociación de la teoría con las experiencias, lo cual posibilita que la información sea recordada con facilidad; El pensamiento crítico se desarrolla a partir del descubrimiento que construye cada estudiante como pensador.

La IEP expone que en la sociedad existen saberes propios de la cultura, y que estos se encuentran en constantes negociaciones con el conocimiento, es decir, la investigación busca en los grupos infantiles y juveniles la unidad y relación de saberes a través de una propuesta metodológica que permite el reconocimiento de los actores sociales que tramitan múltiples métodos investigativos en coherencia con el tipo de problema, (Mejía \& Manjarrés, 2010).

En La IEP se da una novedosa constitución de subjetividad, a partir de la práctica pedagógica brinda la posibilidad de construir nuevos horizontes, donde a partir de la práctica grupal se permite pensar en transformaciones que pueden surgir a partir de la implementación de la misma; los docentes que trabajan con la IEP deben tener claridad de los cambios que se alimentan del ejercicio realizado con los grupos para producir mejoras en su quehacer, (Mejía \& Manjarrés, 2010).

La propuesta de la IEP no es formar científicos, por el contrario, es construir una cultura ciudadana y democrática en ciencia, tecnología e innovación, la educación ha cambiado y como producto de esto, se han integrado nuevos conocimientos tecnológicos, que posibiliten el acceso a la información. La IEP es una de estas, que a través de la justicia educativa y curricular trabaja por construir sociedades justas, a pesar que la IEP no tenga como objetivo principal crear científicos, estos pueden formarse a partir del ejercicio pedagógico de la IEP ya que está promueve en niños y jóvenes con espíritu críticoanalítico, de indagación que les permitirá desarrollar capacidades que contribuyan significativamente a la formación científica, (Mejía \& Manjarrés, 2010).

No obstante, la IEP no solo es ciencia, también está compuesta por el desarrollo de valores y capacidades cognitivas y afectivas, la importancia de la investigación en la sociedad va en aumento, debido a que se encuentran inmersos intereses, que buscan apropiarse de ella como una realidad para construir propuestas que promuevan su apropiación práctica crítica, y que sirva no solo para modernizar, sino, ante todo, para transformar contextos, culturas, epistemologías, y redirigir sus escenarios, (Mejía \& Manjarrés, 2010).

Es así, como las nuevas mediaciones científicas- tecnológicas buscan crear sociedades justas, democráticas y humanas, por esta razón la IEP ha tomado la educación popular y ha rescatado algunos elementos básicos de ellas convirtiéndolos en uno de sus ejes de la propuesta, cuando se realiza un proceso de construcción de una propuesta colectiva, donde se crean grupos de niños, jóvenes, maestros y asesores, con el objetivo de generar otros procesos de educación popular.

En este punto es importante mencionar el modelo pedagógico constructivista, este es un modelo que ha sido concebido como una perspectiva radical que atiende la enseñanza como una actividad critica, mediante este modelo el docente logra hacer una reflexión acerca de su prácticas 
pedagógica; en este modelo los errores son vistos como momentos creativos que le permitirán a los estudiantes ver y conocer otras alternativas, estos suelen generar aprendizaje, puesto que mediante el error los estudiantes deberán arriesgarse e indagar alternativas para la consecución de sus objetivos planteados. Para este modelo de enseñanza el conocimiento no solo debe ser trasmitido por el docente, este debe ser un intercambio de saberes valiéndose de un método de apoyo que le permita al alumno construir su propio saber y conocimiento, (Ministerio, 2012).

El modelo constructivista se caracteriza principalmente por cuatro acciones básicas: apoya la estructura conceptual de cada estudiante, se parte de las ideas y preconceptos que llevan a la clase sobre ciertos temas específicos, este modelo se anticipa al cambio conceptual ocupándose principalmente en la construcción activa de la creación de nuevos conceptos ejercitando la estructura mental de los estudiantes, se confrontan las ideas y pensamientos previos a un proceso de enseñanza y por último se aplican los nuevos conceptos a situaciones concretas con la finalidad de ampliar y divulgar los conocimientos adquiridos, (Ministerio, 2012).

\section{Metodología}

El presente estudio se abordó bajo los lineamientos de la IEP, con un diseño basado en las trayectorias de indagación, utilizando técnicas propias de la investigación cualitativa y cuantitativa necesaria para la recolección de información.

\section{Participantes}

En cuanto a la población beneficiaria, se seleccionó una muestra de cuarenta (40) estudiantes del grado octavo (8) de bachillerato, con edades entre trece (13) y dieciséis (16) años, de la Institución Educativa Departamental Fundación ubicada en el departamento del Magdalena. Estos fueron seleccionados de manera intencional, puesto que presentaron el rendimiento más bajo en la evaluación de competencias, presentando dificultades en el nivel argumentativo.

\section{Técnicas e instrumentos}

Por su parte, en la recolección de datos, se utilizaron técnicas como grupo de discusión y encuestas no estructuradas. Los grupos de discusión se estructuran mediante una conversación grupal con participantes que presenten características similares generando una conversación fluida acerca de un tema en particular, esta técnica es especialmente útil para obtener información acerca de un problema sobre el cual se conoce muy poco, los grupos de discusión pueden ayudar y proporcionar una base para el diseño de preguntas, encuestas, manuales y entrevistas en profundidad.

Estos grupos generalmente generan anécdotas, información sobre patrones, $\mathrm{y}$ visiones internas sobre las normas o actitudes prevalentes (Pérez \& Víquez, 2010). Mientras la encuesta es una técnica que se aplica a una muestra para recoger información sobre creencias, opiniones y actitudes de las personas, identificando normas y patrones de comportamiento para conocer las relaciones entre acontecimientos, (Cruz \& Carmona, 2014).

\section{Procedimiento}

La trayectoria de indagación como metodología, se orienta por la: (a) la diagnosis: revisando qué actividades, herramientas, tiempos, responsables y metas parciales que se propusieron para el primer seg- 
mento o trayecto, así como las funciones de cada uno de los integrantes del grupo para iniciar las actividades de este primer segmento; (b) organizar un archivo y asignar un responsable del mismo: los registros diligenciados deben ser entregados al encargado del archivo, quien será el responsable por su organización y cuidado; (c) recolección de información: consultar diferentes fuentes informativas para conocer los resultados de otras investigaciones sobre el problema de investigación que van a trabajar. En este primer segmento se apoyan con el asesor o líder de investigación, sugiriendo fuentes de información para consultar y los criterios para seleccionar las mismas; (d) elaboración del estado del arte: con la información recolectada elaborar el estado del arte; permite conocer los resultados de otras investigaciones sobre el tema bajo investigación. Además, se puede optar por hablar con investigadores $\mathrm{y}$ conocer experiencias de otros grupos de investigación.

Es importante mantener un registro de las fuentes consultadas y los resultados obtenidos a partir de dichas consultas, como registros audiovisuales, mapas conceptuales, cuestionarios y/o entrevistas, así como los comentarios relevantes del grupo, los cuales pueden compartir con los grupos de la misma línea de investigación a través de un espacio virtual; (e) identificar las técnicas e instrumentos necesarios para el desarrollo de la investigación: con base en el estado del arte, se identifican las técnicas como la entrevista, encuesta, experimentos, mediciones y/o otros a emplear en la investigación. Como instrumentos se tienen en cuenta los materiales e insumos para realizar las pruebas de laboratorio. (f) confrontación de los resultados y propagación de los mismos.

\section{Resultados}

A partir de los resultados obtenidos en el grupo de discusión, los docentes diseñaron una propuesta de integración curricular de la IEP, basada en estrategias pedagógicas dirigidas a fortalecer las competencias argumentativas en los estudiantes del grado octavo de la IED Colombia, dichas estrategias fueron aplicadas, presentando un impacto positivo en la comunidad educativa donde se observó motivación e interés por parte de la población sujeto de estudio, a continuación se detallan las estrategias, (ver tabla 1)

Tabla 1.

Estrategias pedagógicas.

\begin{tabular}{|c|c|c|}
\hline Estrategias & Descripción & Objetivo \\
\hline $\begin{array}{l}\text { Indago y } \\
\text { argumento. }\end{array}$ & $\begin{array}{l}\text { Indagación y argumentación de temática } \\
\text { en ciencias naturales en revistas; Scielo, } \\
\text { Redalyc y Google académico. }\end{array}$ & $\begin{array}{l}\text { Desarrollar las competencias de } \\
\text { investigativas y argumentativas } \\
\text { mediante la indagación. }\end{array}$ \\
\hline $\begin{array}{l}\text { Competencias } \\
\text { lectoras. }\end{array}$ & Léeme, compréndeme y explícame. & $\begin{array}{l}\text { Fortalecer Mediante el ejercicio de } \\
\text { lectura y compresión las competencias } \\
\text { argumentativas en los estudiantes. }\end{array}$ \\
\hline Indagarte. & $\begin{array}{l}\text { Indaga, crea, aprende y argumenta con } \\
\text { arte. }\end{array}$ & $\begin{array}{l}\text { Estimular mediante el arte la } \\
\text { investigación y las competencias } \\
\text { argumentativas en los estudiantes. }\end{array}$ \\
\hline
\end{tabular}

Fuente: elaboración propia (2018). 
A partir del análisis de los resultados presentados en la encuesta se detallan los hallazgos encontrados según cada ítem. En el primer ítem se preguntó; si al momento de leer un texto académico se recordaba con facilidad al día siguiente, para esto el $72.5 \%$ de los estudiantes respondieron de manera afirmativa, infiriendo que la mayoría de los estudiantes logran retener la información con la que han tenido contacto a mediano plazo.

En el segundo ítem de la encuesta se indago acerca de las habilidades que se encontraban más desarrolladas en los estudiantes, para lo cual se preguntó que si al ver en el noticiero una información sobre algún suceso del contexto social se le dificulta explicar detalladamente luego de un par de días frente en esta pregunta los resultados estuvieron notoriamente divididos, donde el $50 \%$ de la población respondió respectivamente a las dos opciones de respuesta. Sin embargo, se piensa que los resultados de esta pregunta pueden estar ligados a los intereses de los estudiantes y a la noticia que se esté presentando.

El tercer reactivo pretendía indagar sobre la comprensión lectora para ello se preguntó; si comprendían con facilidad la información que han leído, en esta pregunta 28 estudiantes respondieron afirmativamente, lo cual corresponde al $70 \%$ de la población encuestada, lo cual implica que estos tendrán facilidad para sustentar lo que han leído argumentando sobre ello, teniendo en cuenta que la comprensión lectora es un proceso que antecede la capacidad argumentativa.

Por su parte el cuarto ítem, buscó hacer una comparación con la pregunta anterior sobre la información que más se le facilita comprender y explicar a los es- tudiantes visual o auditiva por tal razón se les pregunto si comprendían con facilidad los detalles de la información que escuchan. Donde se observó nuevamente una división del $50 \%$ a las dos opciones de respuesta respectivamente. En cuanto al quinto ítem se preguntó si se les dificultaba explicar con sus propias palabras un texto que hayan leído con antelación, con la finalidad de evaluar su retención y argumentación donde se encontró que el 55\% estudiantes no presentan dicha dificultad, lo cual indica que casi la mitad de la población encuestada debe reforzar estas habilidades.

El ítem número seis, busco indagar el nivel de argumentación que poseen actualmente los estudiantes a partir de la intervención de las estrategias, por lo cual se preguntó si se les facilitaba explicar con sus propias palabras una noticia que han escuchado en algún medio de comunicación a lo que el $52.5 \%$ respondió estudiantes que si se les facilitaba explicar con sus propias palabras lo escuchado de las noticias. Mientras el séptimo ítem tenía como objetivo indagar si el problema de argumentación se presentaba para exponer temas académicos o cotidianos para ello en la encuesta se les pregunto que si cuándo se encuentran en una situación problemática se les dificulta explicar los sucesos como acontecieron y se evidencio que los estudiantes no presentan dificultad alguna en expresarse o argumentar temas que ya conocen. Solo el $12.5 \%$ tenía dificultades para exponer situaciones cotidianas.

En el octavo ítem se trataba de indagar si los estudiantes presentaban dificultades en la contrastación de ideas se les pregunto si se les dificultaba contraponer o mostrar la diferencia entre dos o más ideas y según los resultados en su mayoría no presentan problemas 
en sostener una idea observando que el $32.5 \%$ de la población si presenta esta dificultad. Por su parte el noveno ítem indago sobre las ocasiones donde los estudiantes debían leer un texto para comprenderlos en las respuestas de los participantes se evidencia que solo 4 estudiantes leen un texto por una sola vez y lo comprenden el resto de los 36 estudiantes deben leerlo más de una vez para poderlo comprender lo cual representa un $90 \%$ de la población. Mientras el décimo y ultimo ítem se realizó para verificar la respuesta obtenida en el ítem octavo, se preguntó a los estudiantes si se les facilita explicar alguna vivencia, experiencia, suceso, hechos $u$ anécdotas que les hayan sucedido en tu vida cotidiana sin ningún problema a lo que lo el $75 \%$ de los estudiantes respondieron afirmativamente. (Ver tabla 2 ).

\section{Conclusiones}

Los resultados de esta experiencia resultaron en general, mostraron limitaciones, ya que, a partir del diagnóstico realizado por el cuerpo de docentes de la institución, se logró identificar que los estudiantes de octavo grado, presentan dificultades de argumentación, capacidad discursiva, habilidades lectoras, análisis y razonamiento. Esta experiencia permitió a los estudiantes hacer frente a esta problemática, y desarrollar en ellos una serie de habilidades interpersonales y cognitivas de orden superior, además de fomentar una educación integral que aborde competencias necesarias para un óptimo desarrollo social, permitiendo un ejercicio de valores y deberes sustentados en la práctica de una ciudadanía democrática.

Tabla 2

Resultados de la encuesta para evaluar competencias argumentativas.

\begin{tabular}{|c|c|c|}
\hline Pregunta & NO & SI \\
\hline ¿Cuando lees un texto académico lo recuerdas con facilidad el día siguiente? & 11 & 29 \\
\hline $\begin{array}{l}\text { ¿Al ver en el noticiero una noticia sobre algún suceso del contexto social se te } \\
\text { dificulta explicarla detalladamente luego de un par de día? }\end{array}$ & 20 & 20 \\
\hline ¿Comprende con facilidad la información que has leído? & 12 & 28 \\
\hline ¿Comprendes con facilidad los detalles de una información que has escuchado? & 20 & 20 \\
\hline $\begin{array}{l}\text { ¿Se te dificulta explicar con tus propias palabras un texto que has leído con } \\
\text { antelación? }\end{array}$ & 22 & 18 \\
\hline $\begin{array}{l}\text { ¿Se te facilita explicar con tus propias palabras una noticia que has escuchado en } \\
\text { algún medio de comunicación? }\end{array}$ & 19 & 21 \\
\hline $\begin{array}{l}\text { ¿Cuándo te encuentras en una situación problemática se te dificulta explicar los } \\
\text { sucesos como acontecieron? }\end{array}$ & 35 & 5 \\
\hline ¿Se te dificulta contraponer o mostrar la diferencia entre dos o más ideas? & 27 & 13 \\
\hline $\begin{array}{l}\text { ¿Es necesario leer una sola vez un texto para entenderlo y comprenderlo } \\
\text { perfectamente? }\end{array}$ & 36 & 4 \\
\hline $\begin{array}{l}\text { ¿Se te facilita explicar alguna vivencia, experiencia, suceso, hechos u anécdotas que } \\
\text { te hayan sucedido en tu vida cotidiana sin ningún problema? }\end{array}$ & 10 & 30 \\
\hline
\end{tabular}

Fuente: elaboración propia (2018). 
Así, a través de la experiencia realizada, el estudio apunto al fortalecimiento de la competencia argumentativa, puesto que esta presento falencias dentro de las competencias cognitivas. Identificando dificultades para explicar un texto y sustentar actividades que impliquen un nivel básico de razonamiento, por lo anterior el propósito de la investigación fue fortalecer la competencia argumentativa mediante la IEP en educación básica.

Por todo ello, se puede concluir que la propuesta presentada constituye una buena oportunidad tanto para el aprendizaje de los estudiantes, para el enriquecimiento de una formación enfocada en un análisis - crítico e investigativos. La propuesta de integrar la IEP al aula favorece el fortalecimiento de competencias argumentativas, ya que a través de la investigación se fortalecen procesos de pensamiento y razonamiento, permitiendo que los estudiantes organicen, expongan y propongan ideas, contribuyendo a la divulgación del conocimiento, la participación, innovación y competencias comunicativas

\section{Referencias bibliográficas}

Alarcón, D., Cortes, A., \& Rodríguez, V. (2016). Competencias Cognitivas, evaluación constructivista. Redalyc.

Calderón, C., \& Sarmiento, J. (2014). Estrategias pedagógicas para el desarrollo de las competencias interpretativas argumentativas en la lectura y escritura en español en los estudiantes de Educación Básica del Colegio de la Universidad Libre. universidad libre.
Cruz, M., \& Carmona, M. (2014). Competencias argumentativas en estudiantes de educación superior. Revista Internacional de Ciencias Sociales y Humanidades, SOCIOTAM.

Franco, M. (2010). Estrategias de enseñanza y la comprensión lectora. Revista Cultura, Educación, Sociedad-CES, 1(1).

Guzmán, Y., Flores, R., \& Tirado, F. (2013). Desarrollo de la competencia argumentativa en foros de discusión en línea: una propuesta constructivista. Anales de Psicología, vol. 29.

Jiménez, A. (2010). Competencias en argumentación y uso de pruebas: 10 ideas claves. Barcelona, GRAÓ.

Marín, F., Niebles, M., Sarmiento, M., \& Valvuena, S. (2017). Mediación de las tecnologías de la información en la comprensión lectora para la resolución de problemas aritméticos de enunciado verbal. Revista Espacios Vol. $38\left(N^{\circ} 20\right)$.

Mejía, M. R., \& Manjarrés, M. H. (2010). Las pedagogías fundadas en la investigación. Búsquedas en la reconfiguración de la educación. Revista Internacional Magisterio 42: 16-26. Colombia.

Pérez, R., \& Víquez, D. (2010). Los grupos de discusión como metodología adecuada para estudiar las cogniciones sociales. Actualidades en Psicología.

Ramos, A., Herrera, J., \& Ramírez, M. (2010). Desarrollo de habilidades cognitivas con aprendizaje móvil: un estudio de casos. Revista Científica de educomunicaciones.

Rojas, L. R. (2014). Competencias en lenguaje: relaciones entre la escuela y las pruebas de Estado. ICFES. 